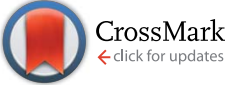

Cite this: RSC Adv., 2016, 6, 76666

Received 2nd June 2016 Accepted 29th July 2016

DOI: $10.1039 / c 6 r a 14368 g$

www.rsc.org/advances

\title{
Nanoscale coordination polymers obtained in ultrasmall liquid droplets on solid surfaces and its comparison to different synthetic volume scales $\uparrow$
}

\author{
E. Bellido, ${ }^{a}$ P. González-Monje, ${ }^{a}$ M. Guardingo, ${ }^{a}$ F. Novio, ${ }^{a}$ A. Sánchez, ${ }^{b}$ M. Montero, ${ }^{b}$ \\ G. Molnar, ${ }^{C}$ A. Bousseksou ${ }^{C}$ and D. Ruiz-Molina*a \\ Synthesis of coordination polymers at femtolitre scales assisted by an AFM tip has become an area of \\ increasing interest due to the astonishing range of implications that derive from it, from gaining basic \\ knowledge of confined reactions in femtolitre droplets to the fabrication of molecular-based devices. \\ However, this field is still in its infancy, a reason why several new basic studies that allow us control over \\ it are highly required. Herein we report the synthesis of $\left[\mathrm{Co}\left(\mathrm{CH}_{3} \mathrm{COO}\right)_{2}\left(\mu-4,4^{\prime}-\right.\right.$ bpy $\left.)\right]$ in femtolitre droplets \\ on surfaces and the results are compared with those obtained for the same reaction at different volume \\ scales.
}

\section{Introduction}

Coordination polymers (CPs), created from limitless combinations of metals and organic building blocks, have been shown to be of relevance in numerous applications, from medicine ${ }^{1}$ or molecular electronics ${ }^{2}$ to catalysis, ${ }^{3}$ among others. Furthermore, the specificity of metal-ligand bonds can be used to thoroughly tune the topology and dimensionality of the polymers. ${ }^{4}$ Therefore, miniaturization of coordination polymers to the nanoscale represents a unique opportunity to assemble a novel class of highly customizable functional materials that combine the rich diversity and properties of coordination complexes and the advantages of nanomaterials. ${ }^{5}$

Recently, direct write AFM-assisted lithography has been shown to be an efficient tool to synthesize, grow and position coordination nanomaterials on surfaces. The main benefit of this technique lays at the possibility to precisely position the desired materials on a surface without the need of any previous functionalization of neither the said materials nor the substrate. ${ }^{6}$ This technique is based on the use of an AFM tip to deliver femtolitre droplets of a solution containing the reactants on a substrate, these femtolitre-sized droplets are subsequently are used as confined nanoreactors where the reaction takes place. By controlling the volume of the delivered droplets,

${ }^{a}$ Catalan Institute of Nanoscience and Nanotechnology (ICN2), CSIC, The Barcelona Institute of Science and Technology, Campus UAB, Bellaterra, 08193 Barcelona, Spain.E-mail: dani.ruiz@icn2.cat

${ }^{b}$ Centro de Electroquímica y Energía Química (CELEQ), Escuela de Química, Universidad de Costa Rica, 11501 2060, San José, Costa Rica

${ }^{c}$ Laboratoire de Chimie de Coordination, CNRS, Université de Toulouse (UPS, INPT), 205, route de Narbonne, 31077 Toulouse Cedex 04, France

$\dagger$ Electronic supplementary information (ESI) available. See DOI: 10.1039/c6ra14368g concentration of reactants and the environmental conditions (mainly but not exclusively temperature and humidity), control over the size and shape of the resulting coordination materials can be achieved.

Our group has successfully used direct write AFM-assisted lithography in the past for the controlled growth of crystals of the well-known metal-organic framework HKUST-1 and spherical polyoxometalate nanostructures. ${ }^{7}$ Carbonell et al. have also described the synthesis and crystal growth of the same HKUST-1 (ref. 8) and other MOFs $^{9}$ using microfluidic writing tools to deposit mixed droplets on a surface. Control over the XY positioning has also allowed for the specific deposition and crystal growth of magnetic coordination complexes on the most sensitive areas of superconducting sensors. ${ }^{10}$ More recently, we reported the in situ synthesis and growth of coordination polymer particles confined inside femtolitre-sized droplets fabricated using AFM-assisted lithography. Beyond achieving precise control over the $X Y$ positioning of the CPs, the experimental methodology employed allowed us to promote the growth of a single nanostructure in each droplet with good monodispersion and tuneable dimensions. ${ }^{\mathbf{1 1}}$

Though pioneering examples have already been described, AFM-assisted synthesis is still in its infancy and a stimulating necessity to face basic scientific challenges exists. Among them, there is a key question that remains open: how is the synthesis and crystallization of coordination materials affected by the translation from the macroscopic to the femtolitre scale? Such information would be crucial to reproduce the enormous library of coordination materials so far reported at the femtolitre scale and therefore to properly fabricate coordination nanomaterials with tuned properties. ${ }^{12}$ However, as far as we know, no previous studies on this matter have been reported. 
a

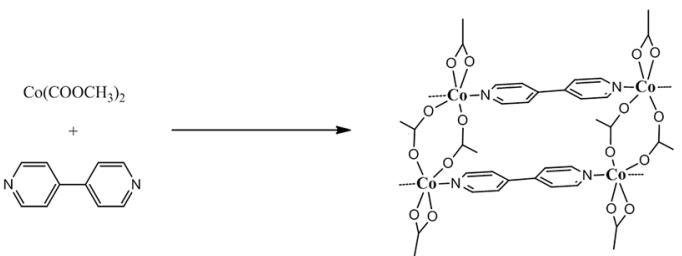

b

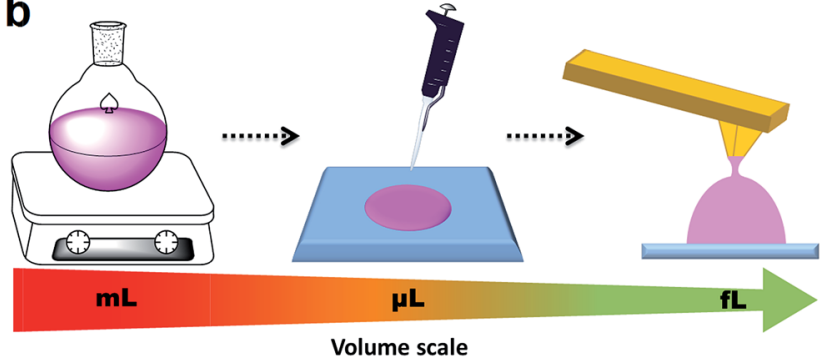

Fig. 1 (a) Schematic of the chemical reaction to obtain coordination polymer 1; (b) representation of the different volume scale for the reaction from bulk synthesis to drop casted on a surface and finally nanostructured with an AFM tip.

Herein we reproduce the synthesis of a model complex, the well-known coordination polymer $\left[\mathrm{Co}\left(\mathrm{CH}_{3} \mathrm{COO}\right)_{2}\left(\mu-4,4^{\prime}\right.\right.$-bpy $\left.)\right]$ (1), in bulk, drop casted on a surface or assisted by an AFM tip, i.e. at the - millilitre $(\mathrm{mL})$, microlitre $(\mu \mathrm{L})$ and femtolitre $(\mathrm{fL})$ scales, respectively. A detailed analysis of the outcome products obtained from these reactions is expected to yield relevant information on the scalability of the synthesis. A schematic representation of this experimental process is shown in Fig. 1.

\section{Experimental}

\subsection{Materials}

Cobalt(II) acetate tetrahydrate ( $\geq 98 \%), 4,4^{\prime}$-bipyridine (bpy) (98\%), glycerol ( $\geq 99 \%)$ and $N, N$-dimethyl-formamide (anhydrous, $99.8 \%$ ) were purchased from Sigma-Aldrich, whereas ethanol absolute (Sps, >99.8\%) and acetone HPLC (>99.9\%) from Fischer. All these materials were not further purified.

\subsection{Substrates}

Silicon substrates bearing a native oxide layer $\left(\mathrm{Si} / \mathrm{SiO}_{2}\right)$ were washed in an ultrasonic bath for $10 \mathrm{~min}$ in acetone, ethanol absolute, and Milli-Q ${ }^{\circledR}$ water and dried by blowing nitrogen gas. These cleaned $\mathrm{Si} / \mathrm{SiO}_{2}$ substrates were also used to prepare gold substrates by thermal evaporation of a $10 \mathrm{~nm}$-thick adhesion layer ( $\mathrm{Ti}$ or $\mathrm{Cr}$ ) and a $40 \mathrm{~nm}$ layer of $\mathrm{Au}$ at a rate of $1 \AA \mathrm{s}^{-1}$ and a base pressure of around $1 \times 10^{-7}$ Torr. Carbon-coated TEM grids, 200 mesh copper grids were purchased from Ted Pella, Inc.

\subsection{Synthesis of $\left[\mathrm{Co}\left(\mathrm{CH}_{3} \mathrm{COO}\right)_{2}\left(\mu-4,4^{\prime}\right.\right.$-bpy $\left.)\right]$ (1) in bulk}

We have selected this complex by using the simple bifunctional ligand 4,4'-bipyridine (bpy) because the stoichiometry of metal : bpy is a critical factor in terms of defining and limiting the possible architectures that can occur in the coordination polymer. ${ }^{13}$ In $15 \mathrm{~mL}$ of DMF, 4,4-bpy $(0.080 \mathrm{~g}, 0.50 \mathrm{mmol})$ was dissolved. After complete dissolution a solution $(2 \mathrm{~mL})$ of $\mathrm{Co}\left(\mathrm{CH}_{3} \mathrm{COO}\right)_{2} \cdot 4 \mathrm{H}_{2} \mathrm{O}(0.062 \mathrm{~g}, 0.25 \mathrm{mmol})$ in DMF was added under stirring. A light pink precipitation formed after a few minutes. After 3 hours, the solution was filtered off, washed with ethanol and dried under vacuum, giving a yield of $80 \%$ based on cobalt acetate. The obtained solid was not soluble in common organic solvents or water. X-ray powder diffraction data for bulk samples were compared with the pattern simulated from the single-crystal structure previously published and proved to correspond to a single phase. Elemental analysis calcd for $\mathrm{C}_{14} \mathrm{H}_{14} \mathrm{O}_{4} \mathrm{~N}_{2}$ Co: C 50.47, H 4.23, N 8.41; found: C 50.74, $\mathrm{H}$ 4.48, N 8.29; IR: 3076 w, 3045 w, 3005 w, 2927 w, 1601 vs, 1556 vs, $1488 \mathrm{~m}, 1436$ vs, 1405 vs, $1335 \mathrm{w}, 1239 \mathrm{w}, 1217 \mathrm{~m}, 1068 \mathrm{~m}$, $1045 \mathrm{~m}, 1006 \mathrm{w}, 935 \mathrm{w}, 884 \mathrm{w}, 816 \mathrm{~s}, 731 \mathrm{w}, 673 \mathrm{~m}, 646 \mathrm{w}, 622 \mathrm{~s}$, $512 \mathrm{w}, 464 \mathrm{~m} \mathrm{~cm}^{-1}$; Raman: $1633 \mathrm{w}, 1612 \mathrm{vs}, 1523 \mathrm{w}, 1328 \mathrm{w}$, 1294 vs, $1246 \mathrm{w}, 1092 \mathrm{~m}, 1019 \mathrm{vs}, 368.1 \mathrm{w}$.

\subsection{Drop casting experiments}

Two different methods were used for drop casting experiments: (a) in situ approach that consisted in the successive drop casting on a silicon surface of two $2 \mu \mathrm{L}$-droplets containing first the cobalt acetate (0.5 mM in DMF) and then the bpy ligand ( $1 \mathrm{mM}$ in DMF); (b) ex situ approach in which the reaction mixture (4 $\mu \mathrm{L}$-droplet) was initially prepared containing the cobalt acetate and bpy (0.5 mM in DMF, $1: 2$ molar ratio) and reacted for five minutes, where the first $60 \mathrm{~s}$ under vigorous stirring. Immediately after, a $4 \mu \mathrm{L}$-droplet of the prepared solution was directly drop casted on the gold surface. After drop casting the samples were left to dry and grow $\mathbf{1}$ in three temperature regimes: (1) room temperature until complete drying, (2) four hours at $50^{\circ} \mathrm{C}$ in the oven, or (3) four hours at room temperature followed by four hours in the oven at $50{ }^{\circ} \mathrm{C}$.

\subsection{AFM assisted synthesis}

Equally to drop casting experiments in situ and ex situ approaches at the femtoliter scale were performed with an Nscriptor ${ }^{\mathrm{TM}}$ DPN System (from NanoInk, Inc.). Environmental conditions of $\sim 75 \%$ and $\sim 45 \%$ of relative humidity for $e x$ situ or in situ reaction respectively, and room temperature were kept constant during all patterning processes with an integrated environmental chamber as part of the Nscriptor ${ }^{\mathrm{TM}}$ DPN System. Tips specially designed for writing purposes were used in all the DPN experiments, specifically, commercial silicon nitride MType Probe Arrays (from NanoInk, Inc.) with a spring constant of $0.5 \mathrm{~N} \mathrm{~m}^{-1}$ and $66 \mu \mathrm{m}$ pitch. To coat the tip, a microfluidic ink delivery chip-based system (Inkwell, from NanoInk, Inc.) was used. The inkwells contain several reservoirs that were filled with the desired ink solutions with the help of a micropipette. Afterwards the ink was transferred to the microwells where the tips were dipped for a given time. The excess of ink coating the tip was removed by bringing the freshly coated tip in contact with gold substrates and creating spots with an average diameter of several micrometers, which quickly decreased in size due to depletion of the ink. After depositing a few micrometer-sized spots, it was possible to start writing uniform dots in a very controllable manner. M-type probe arrays tips were chosen, 
since once coated, allowed creating similar dots of both precursor solutions separately and next performed the mixture between the two solutions placing the reagents together in the same droplet. Substrates were kept in closed vessels saturated with solvent vapour in order to control the evaporation rate of the droplet. The spatial motion of the tip over the surface was perfectly controlled $( \pm 75 \mathrm{~nm} z$ precision and $\pm 100 \mathrm{~nm}$ positional precision over areas as large as $16 \mathrm{~cm}^{2}$ ). Thus a precise positioning of the femtolitre droplets can be programmable and achieved by accurate approach up to the surface.

\subsection{Characterization methods}

Dynamic noncontact mode AFM images were recorded on an Agilent 5500 AFM/SPM microscope. For this purpose, noncontact high-resonance frequency with reflex coating (NCHR) tips were used, which are PPP-NCHR silicon point probes with a spring constant of $\sim 42 \mathrm{~N} \mathrm{~m}^{-1}$ and resonant frequency of $\sim 330$ $\mathrm{kHz}$ (from Nanoandmore). AFM image processing and rendering was performed using Gwyddion data analysis software. SEM images were taken with FEI Quanta 650 FEG operating at $5 \mathrm{kV}$ and SEM MERLIN operating at $2 \mathrm{kV}$. Optical microscopy images were obtained using a Carl Zeiss Axio Observer Z-1 inverted optical microscope. Grazing incidence XRD measurements were performed in a PANalytical X'Pert Pro $\mathrm{X}$-ray diffractometer by using constant grazing incidence $\left(0.5^{\circ}\right)$ and parallel beam optics. Analysed area was about $5 \times 20 \mathrm{~mm}^{2}$. Powder X-ray diffractometry (XRD) was carried out at room temperature on a high-resolution texture diffractometer (PANalytical X'Pert PRO MRD) equipped with a Co-K $\alpha$ radiation source $(\lambda=1.7903 \AA)$ and operating in reflection mode. The solid samples were placed on an amorphous silicon oxide flat plate and measured directly. Raman spectra were acquired at room temperature using a LabramHR microspectrograph (3 $\mathrm{cm}^{-1}$ spectral resolution) coupled to an Andor Technology CCD detector. The $632.8 \mathrm{~nm}$ line of a HeNe laser was used as an excitation source with laser power output of $1 \mathrm{~mW}$. The laser beam was focused on a spot of approximately 1 micrometer in diameter and the Raman signal was collected in a backscattering geometry.

\section{Results and discussion}

\subsection{Bulk synthesis}

The synthesis of the coordination polymer 1 was first carried out in solution following the procedure previously described by Jacobson et al. ${ }^{14}$ Simply, the metal ion source $\left(\left[\mathrm{Co}\left(\mathrm{CH}_{3} \mathrm{COO}\right)_{2}\right]\right.$. $4 \mathrm{H}_{2} \mathrm{O}$ ) and the organic ligand $4,4^{\prime}$-bipyridine (bpy) were mixed under vigorous stirring in $N, N^{\prime}$-dimethylformamide (DMF) under ambient conditions. After several minutes of reaction, a pink-coloured solid precipitated and it was filtered off, washed with ethanol and dried. SEM images confirmed the formation of a highly crystalline solid displaying well-formed flat faces and homogeneous dimensions (Fig. 2a). These crystals exhibited sizes of $2-4 \mu \mathrm{m}$ in length and $0.5-1.0 \mu \mathrm{m}$ in width. The X-ray powder diffraction pattern was in agreement with that obtained from the X-ray diffraction data previously reported. ${ }^{14}$ The

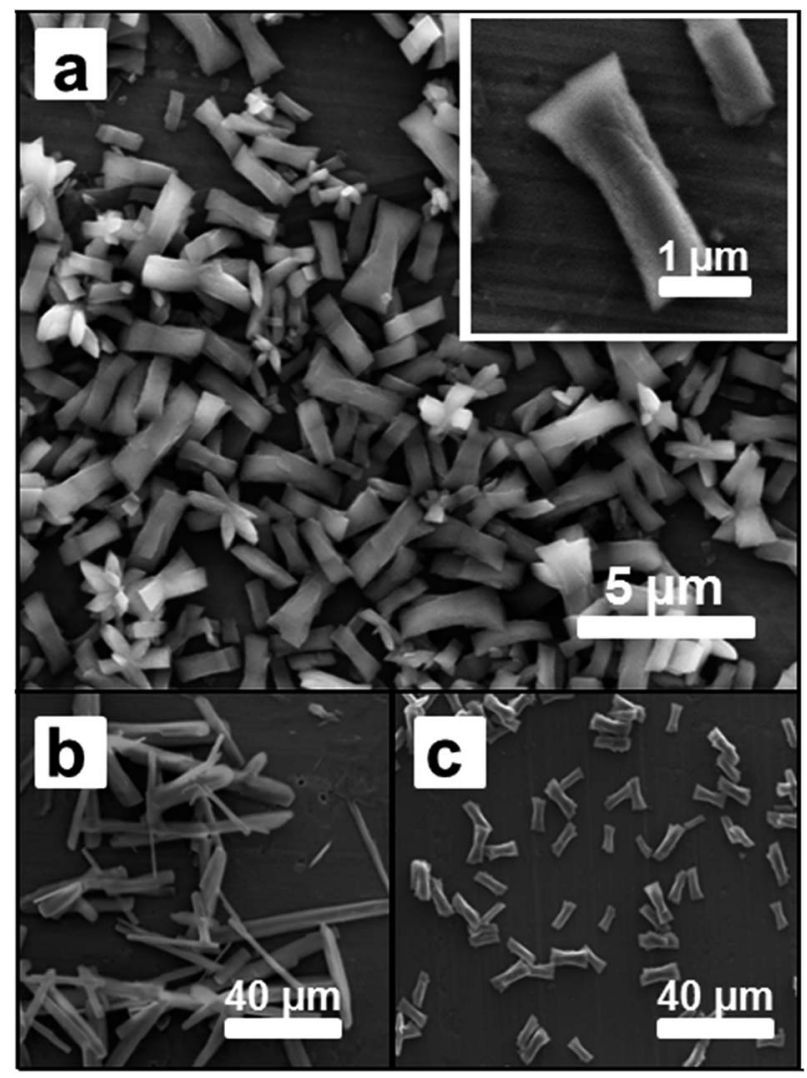

Fig. 2 SEM images of: (a) the $\left[\mathrm{Co}\left(\mathrm{CH}_{3} \mathrm{COO}\right)_{2}\left(\mu-4,4^{\prime}-\right.\right.$ bpy $\left.)\right]$ crystals obtained in DMF under bulk conditions in DMF and (inset) magnification view of a single structure; (b) elongated structures obtained in $\mathrm{EtOH}$; (c) ex situ drop casting procedure in ethanol.

structure consists on the bpy ligands coordinating two cobalt ions and promoting the formation of 1-D polymeric chains while the acetate groups coordinate to cobalt ions from two different chains, forming a ladder-like structure. Moreover the coordination is different for each chain; one anion type uses two oxygen atoms to chelate to cobalt ions forming a fourmembered $\mathrm{Co}-\mathrm{O}-\mathrm{C}-\mathrm{O}$ ring while the other forms a bridge using two oxygen atoms between the cobalt centres in two parallel linear chains (Fig. 1a).

Since our final target was to reproduce the same reaction at the femtolitre scale using AFM-assisted lithography, we had to adapt the reaction conditions to this technique. Briefly, direct write AFM-assisted lithography requires the use of high boiling point and high viscosity solvents to avoid evaporation during the patterning and to ensure a homogeneous coating of the tip and a controlled transference of the material to the surface. In order to adjust those parameters, small percentages of glycerol $(2-5 \% \mathrm{v} / \mathrm{v})$ were added to the reaction solution. FT-IR confirmed no influence of the glycerol addition on the final chemical composition while X-ray powder diffraction confirmed the obtaining of the same crystal phase. Only small morphology variations were found by SEM images (see ESI, S1†).

Interestingly, the small percentage of glycerol added did not affect the final outcome of the reaction though it is well known that solvent nature has an impact in the final morphology and 
dimension of the CP crystals. This has been attributed to the small amount of glycerol added. In fact, when DMF is fully replaced by ethanol more elongated crystals with dimensions between 30 and $60 \mu \mathrm{m}$ in length and 1-5 $\mu \mathrm{m}$ width are obtained (Fig. 2b). Though, worth to mention, drop casting of the same ethanol solution, before precipitation starts to take place, resulted in the obtaining of crystal with the same morphology than those obtained in bulk from DMF (see Fig. 2c). This represents one of the first evidences for the influence of the crystallization process in the morphology of the crystals (vide infra).
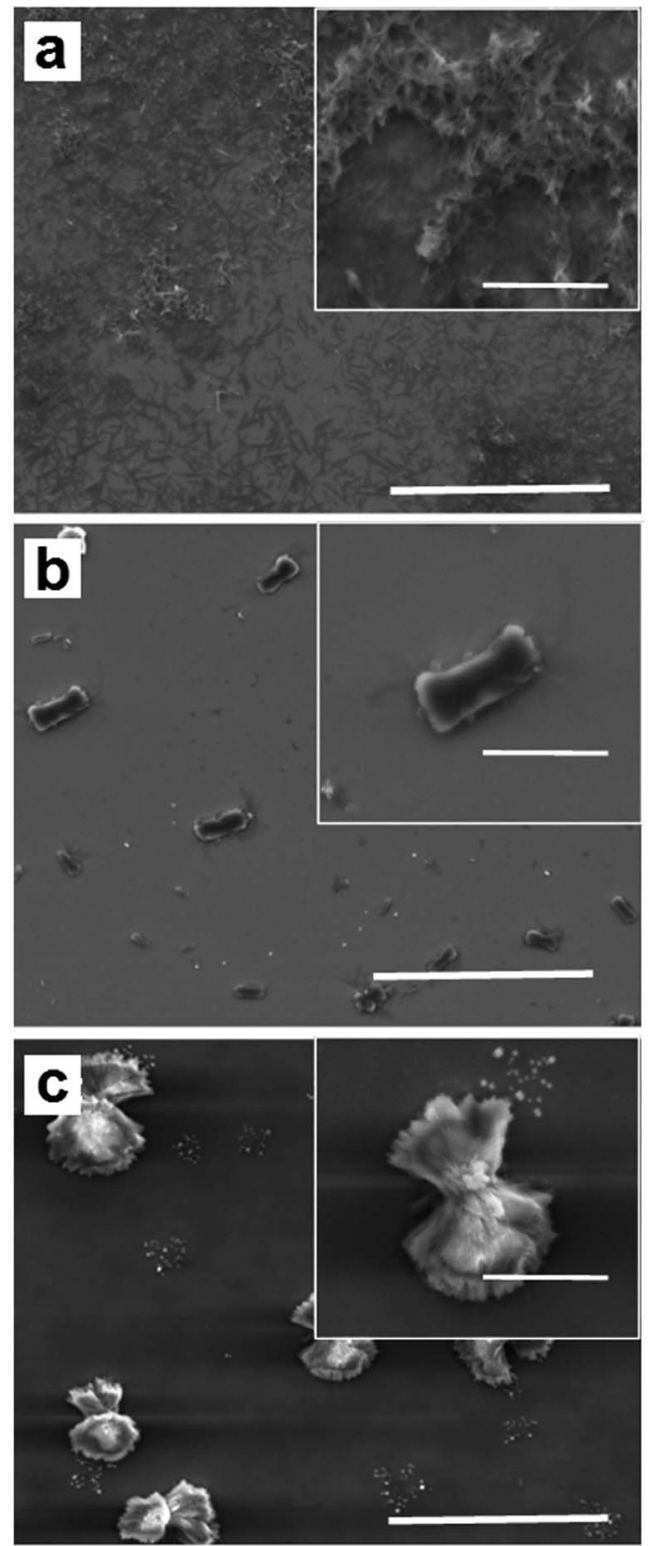

Fig. 3 SEM images of complex 1 obtained upon deposition of $\mu \mathrm{L}$ droplets of a cobalt acetate and bpy (molar ratio $1: 2$ ) DMF solution deposited on a $\mathrm{Si} / \mathrm{SiO}_{2}$ surface and dried following three different drying conditions: (a) room temperature until complete droplet evaporation; (b) $50{ }^{\circ} \mathrm{C}$ for four hours; and (c) combined drying: $4 \mathrm{~h}$ at room temperature and $4 \mathrm{~h}$ at $50^{\circ} \mathrm{C}$. Scale bar represents $10 \mu \mathrm{m}$. Inset: magnification of the structures where the scale bar stands for $3 \mu \mathrm{m}$.

\subsection{Drop casting}

A solution of cobalt acetate and bpy (molar ratio $1: 2$ ) in DMF was prepared and left to react for one minute under vigorous stirring and four additional minutes without stirring. The reaction mixture ( $4 \mu \mathrm{L}$-droplet) was immediately transferred to a $\mathrm{Si} / \mathrm{SiO}_{2}$ surface before the precipitation of the coordination material occurred. In order to find the right conditions to obtain similar microstructures to those observed in bulk, crystallization of $\mathbf{1}$ was induced following three different drying conditions: (i) slow droplet evaporation at room temperature until complete solvent evaporation; (ii) $50{ }^{\circ} \mathrm{C}$ for four hours; and (iii) combined drying: $4 \mathrm{~h}$ at room temperature and $4 \mathrm{~h}$ at $50{ }^{\circ} \mathrm{C}$ (for more details see Experimental section and ESI, S2 $\dagger$ ). Representative SEM images of the different structures obtained after each one of the treatments are shown in Fig. 3. As can be seen there, a relevant influence of the drying process on the final morphology can be found. Bar-like structures were observed when substrates were left to dry at room temperature (Fig. 3a), whilst structures obtained upon heating up to $50{ }^{\circ} \mathrm{C}$ showed flat faces with defined edges, reminiscent of those obtained as bulk materials (Fig. 3b). An intermediate situation was observed in samples subjected to a two-step drying process (first room temperature, then $50{ }^{\circ} \mathrm{C}$ ) where the objects have the shape of bundle with well-defined structure, broader in the edges (Fig. 3c). Moreover, XRPD data corroborated that the crystalline phase of the resulting structures was identical to the one obtained for the bulk microcrystalline material. As a representative example, XRPD data of the structures obtained after heating a deposited substrate for fours hour at $50{ }^{\circ} \mathrm{C}$ are shown in Fig. 4. Interestingly, similar morphologies are obtained following the same deposition process and thermal treatment but changing the molar ratio from $1: 2$ to $1: 1$ (see ESI, S3†).

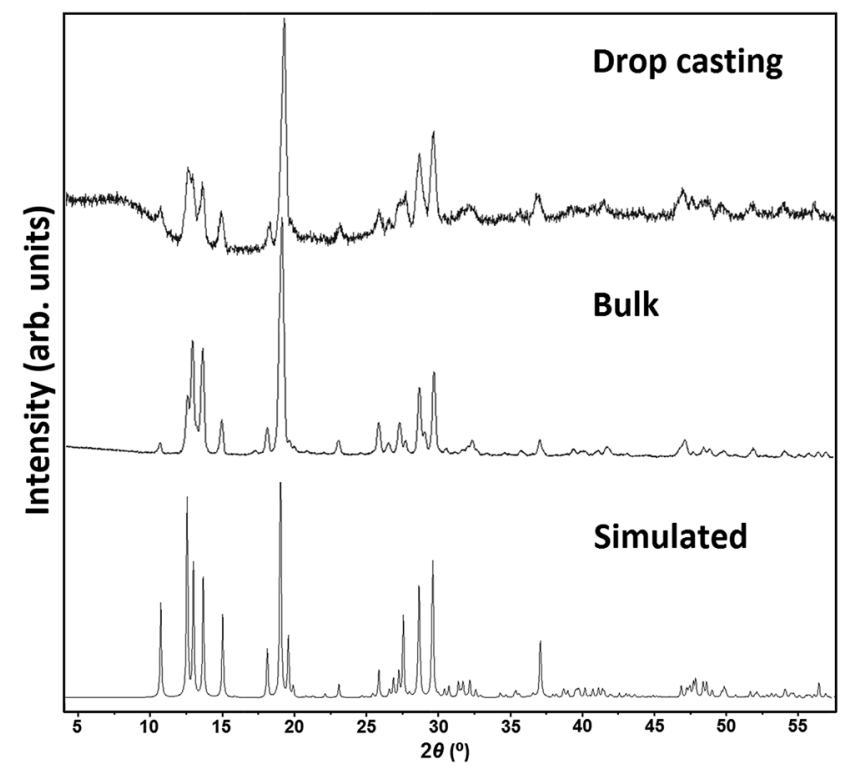

Fig. 4 XRPD patterns of $\left[\mathrm{Co}\left(\mathrm{CH}_{3} \mathrm{COO}\right)_{2}\left(\mu-4,4^{\prime}-\right.\right.$ bpy $\left.)\right]$ obtained by drop casting and in bulk with the simulation pattern for the corresponding solved structure. ${ }^{14}$ 
To confirm that the structures shown in Fig. 4 were obtained right after depositing the droplets, and not during the manipulation process, the reaction was also carried out in situ. For this, $2 \mu \mathrm{L}$-droplets of a cobalt acetate solution (in DMF) and bpy ligand (in DMF) with two different molar ratios $1: 2$ and $1: 1$ were successively casted onto the target surface, creating $\mu \mathrm{L}$ sized reaction vessels where the coordination reaction occurred. After mixing both droplets, different drying conditions previously used: (i) room temperature until complete droplet evaporation; (ii) $50{ }^{\circ} \mathrm{C}$ for four hours; and (iii) combined drying: $4 \mathrm{~h}$ at room temperature and $4 \mathrm{~h}$ at $50{ }^{\circ} \mathrm{C}$. Representative SEM images of the different structures obtained after each one of the treatments are shown in ESI, S4 and S5. $\dagger$ The images reveal microstructures similar to those obtained by the ex situ process, confirming the consistency of the approach. Moreover, XRPD data corroborated the same crystalline phase for the all the structures obtained through both approaches, which in turn were identical to the XRPD data obtained for the bulk microcrystalline material (see ESI, S6†).

Finally, as previously done for the bulk studies, the reaction was repeated now with a small percentage of glycerol $(2-5 \% \mathrm{v} / \mathrm{v})$. FT-IR confirmed no influence of the glycerol addition on the final chemical composition while XRPD revealed also the consistency of the crystal phase.

\subsection{Tip-assisted synthesis}

Finally, the synthesis of $\mathbf{1}$ was carried out in femtolitre-sized reaction vessels fabricated using AFM-assisted lithography. Two different approaches, in situ and ex situ, were followed as schematically shown in Fig. 5 .

In the in situ approach (Fig. 5a), two consecutive AFM tips of an array were differently coated with the organic ligand (tip 1) and the metal salt solution (tip 2). Then, the coated tips were used to directly transfer the solutions to the same position of the surface and mixed droplets were obtained where the coordination reaction and crystallization process occurred. In the ex situ approach (Fig. 5b) the reagents were previously mixed in a vial and the mixture was immediately patterned on the surface to confine the crystal growth of 1 . Experimental details for each approach are given next.

For the in situ experiments ink solutions of both molecular precursors, a $0.1 \mathrm{mM}$ bpy solution and a $0.1 \mathrm{mM}\left[\mathrm{Co}\left(\mathrm{CH}_{3}-\right.\right.$ $\left.\mathrm{COO})_{2}\right] \cdot 4 \mathrm{H}_{2} \mathrm{O}$ solution in DMF containing glycerol $(5 \% \mathrm{v} / \mathrm{v})$ were prepared and transferred to adjacent channels of a microfluidic ink delivery system (inkwell) (see Experimental section). Afterwards, M-type pen arrays (66 $\mu \mathrm{m}$ pitch) were dipped in each solution to obtain two tips functionalized with the ligand (tip 1) and the metal salt (tip 2) located side by side.

The coated tips were then brought into contact with the substrate to deposit femtolitre-sized droplets of the two separate solutions. Finally, a lateral translation of the tips $(66 \mu \mathrm{m})$ allowed us to deliver droplets of the second reagent over the first one. In this way femtolitre-sized droplets of the reacting mixture were fabricated in situ to act as nanoreactors. Importantly, the experiments were carried out under high relative humidity conditions $(\sim 75 \%)$ and at room temperature to avoid the fast

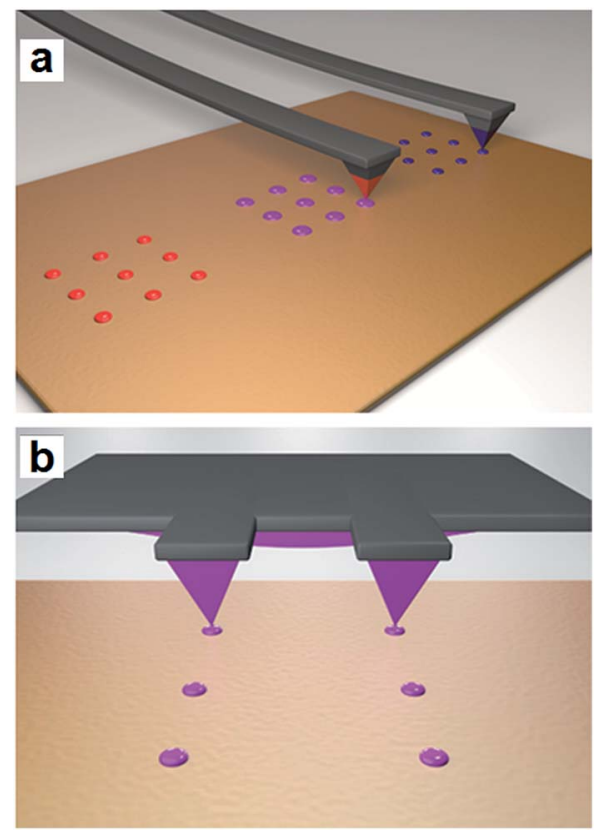

Fig. 5 Schematic representation of: (a) the reaction of $\mathrm{Co}\left(\mathrm{CH}_{3} \mathrm{COO}\right)_{2}$ (blue color, tip 1) with bpy (red color, tip 2) through superimposed lithographies. First dot-like feature arrays were obtained over the surface with each one of the inks and after a lateral movement of the tips a second lithography is performed over the first one, resulting in the mixture of the two inks (purple color); (b) the reagents are previously mixed in a vial (pink color) and the reaction mixture is deposited on the surface during the early stages of the reaction, before the growth and precipitation of complex 1 takes place.

evaporation of the solvent during the lithographic process. Immediately after the deposition the sample was stored inside an environmental chamber for $12 \mathrm{~h}$ and the relative humidity was allowed to gradually decrease to ambient conditions. As can be seen in Fig. 6, examination of the dots after complete solvent

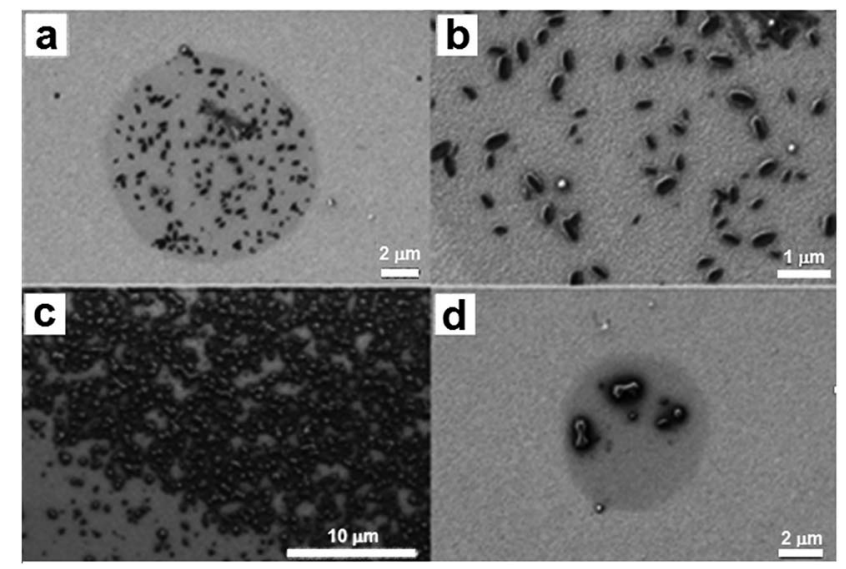

Fig. $6\left[\mathrm{Co}\left(\mathrm{CH}_{3} \mathrm{COO}\right)_{2}\left(\mu-4,4^{\prime}-\right.\right.$ bpy $\left.)\right]$ obtained by AFM-assisted lithography on surfaces through in situ deposition method. (a)-(d) SEM images of the structures obtained inside different dots deposited on surfaces and magnification view. The elongated shapes were the most common but other structures such as bundle-like could be observed (d). 
evaporation by SEM revealed the presence of several elongated structures with loosely defined faces and round edges (mean size: $338 \pm 49 \mathrm{~nm}$ in length and $169 \pm 26 \mathrm{~nm}$ in width, aspect ratio $2: 1$ ).

It is important to note that the order of addition of the reagents in the in situ approach had no important influence on the morphology of the obtained crystals. Moreover, the use of the different drying conditions previously used resulted in a material of undefined shape whereupon was difficult to reproduce those obtained in bulk and by drop casting.

For the ex situ experiments equivalent volumes of $0.5 \mathrm{mM}$ solutions of both reagents in DMF containing glycerol $(2 \% \mathrm{v} / \mathrm{v})$ were mixed under vigorous stirring. The mixed solution was transferred to a microfluidic ink delivery system and M-type pen arrays were functionalised with the mixture and brought into contact with a gold substrate under controlled environmental conditions ( $45 \%$ relative humidity and room temperature). After the fabrication of the dot-like features, the sample was kept inside the environmental chamber for $12 \mathrm{~h}$ to reach environmental conditions. As a representative example, Fig. 7 shows the SEM image of one dot-like feature array composed of 12 features $(2 \times 6$ dot array). SEM micrographs showed the growth of well-defined, elongated crystals with rounded edges inside each droplet (mean size: $265 \pm 21 \mathrm{~nm}$ in length and $137 \pm 11$ $\mathrm{nm}$ in width, long and short axis respectively, aspect ratio $2: 1$ ). The resulting structures were also characterized by AFM and a $3 \mathrm{D}$ reconstruction of the crystalline material was obtained (Fig. 7d). Worth to mention, the size and morphology was independent of the deposited volume and similar structures were obtained when the material was deposited on different substrates, such as gold, $\mathrm{Si} / \mathrm{SiO}_{2}$ substrates and amorphous carbon-coated TEM grids. Representative TEM images of the resulting microstructures upon femtolitre deposition on the

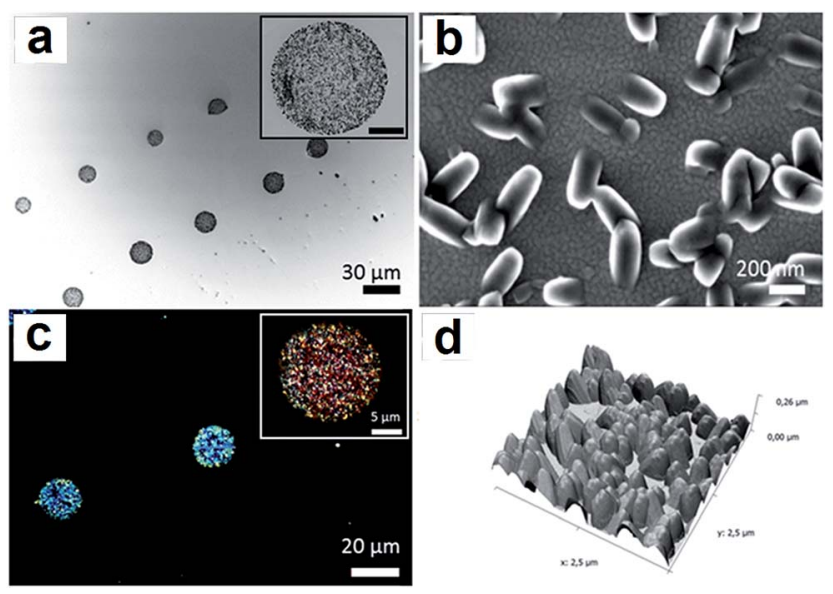

Fig. $7\left[\mathrm{Co}\left(\mathrm{CH}_{3} \mathrm{COO}\right)_{2}\left(\mu-4,4^{\prime}\right.\right.$-bipy $\left.)\right]$ obtained by AFM-assisted lithography on surfaces through ex situ deposition method. (a) SEM image of series of microscale dots on Au surface and magnification view of a single dot (inset). (b) SEM image of detailed morphology of crystalline material. (c) Optical images obtained by polarized light of microscale dots and magnification of a single dot (inset). (d) AFM topographical image of detailed topology of the deposited material by 3D reconstruction.

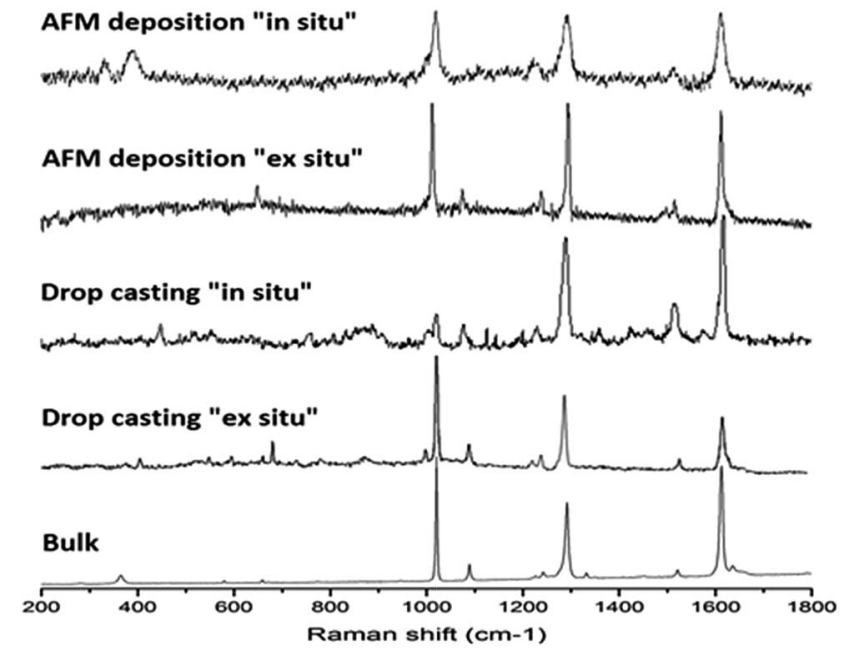

Fig. 8 Comparison of $\mu \mathrm{RS}$ spectra of the resulting material obtained in bulk and deposited in situ on gold surfaces through drop casting or AFM assisted lithography. The concordance of the patterns corroborates the maintenance of the chemical entity using the different methodologies.

TEM grids are shown in the ESI, S7. $\dagger$ In this case, flat faces of homogeneous dimensions were also obtained. The crystalline nature of the obtained structures was confirmed by the use of polarized light (Fig. 7c). However, the corresponding XRP diffractogram could not be obtained due to the small amount of material present in the sample, which was below the sensibility level of the equipment.

Finally, microRaman spectroscopy ( $\mu \mathrm{RS}$ ) was used to confirm that the tip-assisted reaction also leads to the formation of complex 1. Since the laser beam of this technique is focused to a micron-size and can analyse with a $\mu \mathrm{m}^{3}$ sample volume, $\mu \mathrm{RS}$ offers the required high spatial resolution and sensitivity. The spectra were recorded in the $1800-200 \mathrm{~cm}^{-1}$ range for AFM assisted microstructures obtained following both the in situ and ex situ approaches. For comparison purposes, $\mu \mathrm{RS}$ of the samples obtained by drop casting (both in situ and ex situ) as well as bulk samples were also analysed. The spectra of all five samples were found to be in excellent agreement with bands at 1027, 1290, and 1525-1615 $\mathrm{cm}^{-1}$, corresponding to the ring breathing, the $\mathrm{CH}$ bending, and bpy ring stretching vibrations, respectively. These signals are in agreement with previous Raman studies for Co-pyridine and Co-bipyridine complexes (Fig. 8). ${ }^{15}$

\section{Conclusions}

We have reported the controlled synthesis of the coordination polymer 1 inside femtolitre reactors deposited on surfaces assisted by an AFM tip. This was achieved either by direct mixing of femtolitre droplets of the reagents through sequential delivery on a surface or by pre-mixing them on a vial and immediately transferring the mixture with an AFM tip before any crystallization starts to take place. Well-defined, elongated crystals with rounded edges a few hundred nanometers length 
and a few tens of nanometres width were obtained for both the in situ and ex situ approaches. These studies have demonstrated that to obtain microstructures with similar morphology to the crystals obtained in bulk, the substrates must be kept under an environmental chamber along the whole process in order to control the evaporation rate of the droplet so a proper crystallization process takes place. This turned out to be crucial since droplets at this scales may evaporate very fast leading to an undesired premature precipitation. In fact, all the experiments carried out done without taking into account such considerations yielded amorphous materials lacking any defined shape. This could be also the origin for the round edged nature of the resulting elongated crystals.

Interestingly, to obtain similar microstructures of complex 1 from drop casting, heating of the substrates up to $50{ }^{\circ} \mathrm{C}$ for four hours has been required. Otherwise, longer needles and/or bundles of them are obtained. Considering that the experimental conditions (solvent nature, concentration or substrate) are the same, such differential behaviour may be tentatively attributed to the differential evaporation of microliter and femtolitre droplets on the surface and possibly to confinement effects. We expect that the results reported will play a significant role in the advancement of synthesis and crystallization in ultrasmall droplets.

\section{Acknowledgements}

M. G. thanks the CSIC for a predoctoral grant (JAEpre). This work was supported by project MAT2015-70615-R from the Spanish Government and by FEDER funds. ICN2 acknowledges support from the Severo Ochoa Program (MINECO, Grant SEV2013-0295).

\section{Notes and references}

1 (a) F. Novio, J. Simmchen, N. Vázquez-Mera, L. Amorín-Ferré and D. Ruiz-Molina, Coord. Chem. Rev., 2013, 257, 28392847; (b) P. Horcajada, R. Gref, T. Baati, P. K. Allan, G. Maurin, P. Couvreur, G. Férey, R. E. Morris and C. Serre, Chem. Rev., 2012, 112, 1232-1268; (c) J. Della Rocca, D. Liu and W. Lin, Acc. Chem. Res., 2011, 44, 957-968.

2 (a) T. Tezgerevska, K. G. Alley and C. Boskovic, Coord. Chem. Rev., 2014, 268, 23-40; (b) A. Bousseksou, G. Molnár, L. Salmon and W. Nicolazzi, Chem. Soc. Rev., 2011, 40, 3313-3335; (c) E. Evangelio and D. Ruiz-Molina, Eur. J. Inorg. Chem., 2005, 2957-2971.

3 S. Kitagawa, R. Kitaura and S. Noro, Angew. Chem., Int. Ed., 2004, 43, 2334-2375.

4 (a) M. Oh and C. A. Mirkin, Nature, 2005, 438, 651-654; (b) Ch. Janiak, Dalton Trans., 2003, 2781-2804; (c) S. R. Batten, Curr. Opin. Solid State Mater. Sci., 2001, 5, 107-114.

5 (a) A. M. Spokoyny, D. Kim, A. Sumrein and C. A. Mirkin, Chem. Soc. Rev., 2009, 38, 1218-1227; (b) W. Lin,
W. J. Rieter and K. M. L. Taylor, Angew. Chem., Int. Ed. Engl., 2009, 48, 650-658.

6 (a) M. Guardingo, M. J. Esplandiu and D. Ruiz-Molina, Chem. Commun., 2014, 50, 12548-12551; (b) B. Radha, G. Liu, D. J. Eichelsdoerfer, G. U. Kulkarni and C. A. Mirkin, ACS Nano, 2013, 7, 2602-2609; (c) G. Arrabito, S. Reisewitz, L. Dehmelt, P. I. Bastiaens, B. Pignataro, H. Schroeder and C. M. Niemeyer, Small, 2013, 9, 4243-4249; (d) A. Hernandez-Santana, E. Irvine, K. Faulds and D. Graham, Chem. Sci., 2011, 2, 211-215; (e) F. Brinkmann, M. Hirtz, A. M. Greiner, M. Weschenfelder, B. Waterkotte, M. Bastmeye and H. Fuchs, Small, 2013, 9, 3266-3275.

7 E. Bellido, S. Cardona-Serra, E. Coronado and D. RuizMolina, Chem. Commun., 2011, 47, 5175-5177.

8 C. Carbonell, I. Imaz and D. Maspoch, J. Am. Chem. Soc., 2011, 133, 2144-2147.

9 C. Carbonell, K. C. Stylianou, J. Hernando, E. Evangelio, S. A. Barnett, S. Nettikadan, I. Imaz and D. Maspoch, Nat. Commun., 2013, 4, 2173.

10 (a) E. Bellido, P. González-Monje, A. Repollés, M. Jenkins, J. Sesé, D. Drung, T. Schurig, K. Awaga, F. Luis and D. Ruiz-Molina, Nanoscale, 2013, 5, 12565-12573; (b) M. J. Martínez-Pérez, E. Bellido, R. De Miguel, J. Sesé, A. Lostao, C. Gómez-Moreno, D. Drung, T. Schurig, D. Ruiz-Molina and F. Luis, Appl. Phys. Lett., 2011, 99, 1013; (c) N. Domingo, E. Bellido and D. Ruiz-Molina, Chem. Soc. Rev., 2012, 41, 258-302; (d) E. Bellido, N. Domingo, I. Ojea-Jiménez and D. Ruiz-Molina, Small, 2012, 8, 14651491.

11 M. Guardingo, P. González-Monje, F. Novio, E. Bellido, F. Busqué, G. Molnár, A. Bousseksou and D. Ruiz-Molina, ACS Nano, 2016, 10, 3206-3213.

12 S. Diring, S. Furukawa, Y. Takashima, T. Tsuruoka and S. Kitagawa, Chem. Mater., 2010, 22, 4531-4538.

13 (a) L. R. MacGillivray, S. Subramanian and M. J. Zaworotko, J. Chem. Soc., Chem. Commun., 1994, 1325-1326; (b) R. W. Gable, B. F. Hoskins and R. Robson, J. Chem. Soc., Chem. Commun., 1990, 1677-1678; (c) M. Fujita, Y. J. Kwon, S. Washizu and K. Ogura, J. Am. Chem. Soc., 1994, 116, 1151-1152; (d) R. Robson, B. F. Abrahams, S. R. Batten, R. W. Gable, B. F. Hoskins and J. Liu, Supramolecular Architecture, 1992, 19, 449; (e) L. Carlucci, G. Ciani, D. M. Proserpio and A. Sironi, J. Chem. Soc., Chem. Commun., 1994, 2755-2756; ( $f$ ) S. Subramanian and M. J. Zaworotko, Angew. Chem., Int. Ed. Engl., 1995, 34, 2127-2129.

14 J. Lu, C. Yu, T. Niu, T. Paliwala, G. Crisci, F. Somosa and A. J. Jacobson, Inorg. Chem., 1998, 37, 4637-4640.

15 (a) D. Y. Wu, Y. Xie, B. Ren, J. W. Yan, B. W. Mao and Z. Q. Tian, PhysChemComm, 2001, 18, 1-3; (b) N. Y. Nia, P. Farahani, H. Sabzyan, M. Zendehdelc and M. Oftadeh, Phys. Chem. Chem. Phys., 2014, 16, 11481-11491. 